\title{
Molecular basis of dimerization of initiator caspase was revealed by crystal structure of caspase-8 pro-domain
}

\author{
Hyun Ho Park ${ }^{1}$
}

Received: 2 July 2018 / Accepted: 28 August 2018 / Published online: 11 September 2018

(c) ADMC Associazione Differenziamento e Morte Cellulare 2018

\begin{abstract}
The assembly of death-inducing signaling complex (DISC) for activation of initiator caspase is a key step for the receptormediated apoptosis signaling. Many death effector domain (DED)-containing proteins are involved in DISC assembly and controlling. One of the main DISC component, caspase-8, contains DED and DED-mediated dimerization and oligomerization in the DISC is critical for the activation of this initiator caspase. There have been intensive studies to understand DED-mediated dimerization and oligomerization for the DISC assembly but no clear answer has been provided and there are many controversial arguments. Here, we suggested novel dimerization process of tandem DED of caspase- 8 with crystallographic study.
\end{abstract}

\section{Introduction}

Caspases, a family of cysteine proteases that cleave specifically after aspartic acid residues, are involved in the apoptosis and inflammation signaling pathways [1-3]. Based on their roles, caspases can be divided into two classes: initiator caspases, including caspase-1, -8 , and -10 , and effector caspases, including caspase-3 and -7. Initiator caspases are synthesized as zymogens, which possess prodomains, and are auto-processed upon dimerization through recruitment to oligomeric signaling complexes [4]. Caspase8 is the main initiator caspase in death receptor-mediated apoptosis. It was discovered as a component of the deathinducing signaling complex (DISC), which contains a death receptor, FADD (Fas-associated protein with death domain), and caspase-8 [5]. Zymogen of caspase-8, also called procaspase- 8 , consists of a pro-domain harboring tandem death effector domains (DEDs) followed by a

Edited by V. Dixit

Electronic supplementary material The online version of this article (https://doi.org/10.1038/s41418-018-0200-x) contains supplementary material, which is available to authorized users.

Hyun Ho Park xrayleox@cau.ac.kr

1 College of Pharmacy, Chung-Ang University, Seoul 06974, South Korea caspase domain. DISC formation for caspase-8 activation depends on the protein interaction domain called the death domain superfamily, which contains four subfamily members, including death effector domain (DED), death domain (DD), caspase-recruiting domain (CARD), and Pyrin domain (PYD) [6]. During the extrinsic apoptosis process, procaspase- 8 is recruited to the death receptor through the bipartite adaptor protein FADD [7]. The cytosolic component of the death receptor contains DD. FADD contains DD at the $\mathrm{N}$ terminus and DED at the $\mathrm{C}$ terminus. Death receptor interacts with FADD via DD: DD interactions, and caspase-8 interacts with FADD via DED: DED interactions. Upon DISC formation, caspase- 8 acquires proximity that can induce dimerization and auto-proteolytic processing [8]. Although the main function of procaspase- 8 is to induce apoptosis through death receptors, many studies have shown that it can regulate necroptosis and nuclear factor- $\mathrm{\kappa B}$ signaling through various receptor signaling events, including $\mathrm{Fc}$ and Toll-like receptors in $\mathrm{T}, \mathrm{B}$, and natural killer cells [9-12]. Although the enzymatic function of caspase- 8 is critical for apoptosis, the adaptor function has also been emphasized in immune cell signaling [12].

Caspase- 8 tandem DEDs and the process of dimerization or oligomerization for DISC assembly have been intensively studied and discussed [13-17]. In addition, studies have investigated how DED-containing proteins, such as FADD, FLIP, PEA-15, DEDD, and caspases, can interact with each other via DED-DED or DED-tandem DED interactions [18]. Despite these studies, there is no clear 
evidence, and many controversial oligomerization models have been proposed $[16,19]$. Therefore, in this study, we introduced the structure of intact tandem DEDs of caspase8. Our structure showed that tandem DEDs of caspase- 8 dimerized via a novel domain-swapped strategy, which support the previous finding that the FL motif at DED2, not in DED1, is critical for the dimerization and further activation of caspase-8 in DISC.

\section{Experimental procedures}

\section{Sequence alignment}

The amino acid sequences of DED domains were analyzed using ClustalW2 (http://www.ebi.ac.uk/Tools/msa/clustalw2/).

\section{Protein expression and purification}

Pro-domain of caspase-8 (amino acid residue: 1-178) was expressed in Escherichia coli. Full-length human caspase-8 (Genebank ID: BAB32555.1) was amplified by PCR using gene-specific primers containing NdeI and XhoI sites. PCR fragments were subsequently digested and ligated into the pET24a vector containing the C-terminal hexa-histidine tag. The sequences of cloned genes were verified by DNA sequencing. The resulting plasmid was transformed into BL21 (DE3) E. coli-competent cells. After this, the cells were plated onto LB (Luria-Bertani) media and incubated for $24 \mathrm{~h}$ at $310 \mathrm{~K}$. Next, individual colonies were inoculated into $5 \mathrm{~mL} \mathrm{LB}$ media and incubated overnight at $310 \mathrm{~K}$ with shaking. Cultured cells were then moved to $1 \mathrm{~L} \mathrm{LB}$ media and incubated for $4 \mathrm{~h}$ at $310 \mathrm{~K}$ until optical density reached to 0.7 with shaking. After this, the expression was induced by treating the bacteria with $0.25 \mathrm{mM}$ isopropyl $\beta$-D-thiogalactopyranoside (IPTG) for $18 \mathrm{~h}$ at $293 \mathrm{~K}$. Following induction, the bacteria were collected, resuspended, and lysed by sonication in $50 \mathrm{~mL}$ lysis buffer $(20 \mathrm{mM}$ Tris- $\mathrm{HCl}$ $\mathrm{pH} 7.9,500 \mathrm{mM} \mathrm{NaCl}, 10 \mathrm{mM}$ imidazole). The bacterial lysate was subsequently centrifuged at $16,000 \mathrm{rpm}$ for 30 min at $277 \mathrm{~K}$. After this, the supernatant was applied to a gravity-flow column (Bio-Rad) packed with $1.5 \mathrm{~mL}$ of NiNTA affinity resin (Qiagen). The unbound bacterial proteins were subsequently removed from the column using $50 \mathrm{~mL}$ washing buffer $(20 \mathrm{mM}$ Tris- $\mathrm{HCl} \mathrm{pH} 7.9,500 \mathrm{mM} \mathrm{NaCl}$, $25 \mathrm{mM}$ imidazole). The target protein was subsequently eluted from the column using an elution buffer $(20 \mathrm{mM}$ Tris- $\mathrm{HCl} \mathrm{pH} 7.9,500 \mathrm{mM} \mathrm{NaCl}, 250 \mathrm{mM}$ imidazole). Fractions containing more than $90 \%$ homogenous proteins upon sodium dodecyl sulfate-polyacrylamide gel electrophoresis (SDS-PAGE) were then selected and combined. After this, the protein purity was further improved using a Superdex 200 gel-filtration column 10/30 (GE Healthcare) pre-equilibrated with a solution of $20 \mathrm{mM}$ Tris- $\mathrm{HCl} \mathrm{pH} 8.0$ and $150 \mathrm{mM} \mathrm{NaCl}$.

\section{Crystallization and data collection}

The initial conditions for crystallization were screened at $293 \mathrm{~K}$ by the hanging-drop vapor-diffusion method using screening kits from Hampton Research and the deCODE Biostructure Group. Initial crystals were grown on a siliconized cover slip by equilibrating a mixture containing 1 $\mu \mathrm{L}$ of protein solution $(6-7 \mathrm{mg} / \mathrm{mL}$ protein in $20 \mathrm{mM}$ Tris pH 8.0 and $150 \mathrm{mM} \mathrm{NaCl}$ ) and $1 \mu \mathrm{L}$ of reservoir solution containing $2.0 \mathrm{M}$ ammonium sulfate, $0.2 \mathrm{M} \mathrm{NaCl}$, and 0.1 $\mathrm{M}$ cacodylate $\mathrm{pH} 6.5$ against $0.4 \mathrm{~mL}$ of reservoir solution. The initial crystals from the screening were sufficient for collecting the data set. Crystals reached to maximum dimensions of $0.3 \times 0.3 \times 0.3 \mathrm{~mm}$ in 3 days. The diffraction data set at $2.1 \AA$ resolution was collected at Pohang Accelerator Laboratory (PAL) $5 \mathrm{C}$ beamline in South Korea and processed using the HKL2000 package [20] (Table S1).

\section{Structural determination and analysis}

The molecular replacement (MR) phasing method was conducted with Phaser [21] using the F122G/L123G double mutant form of caspase-8 (PDBid: 5JQE) [17] as a search model. The initial solution model was extended and completed by iterative manual building and refinement in Coot [22] and PHENIX Refine [23], respectively. The quality of the model was checked using PROCHECK [24]. Molecular structure images were generated using the PyMOL Molecular Graphics System, Version 1.3 Schrödinger, LLC.

\section{Multi-angle light scattering (MALS)}

The absolute molar mass of the caspase- 8 tandem DEDs (1-178) was determined by MALS. Briefly, the target protein was loaded onto a Superdex $200 \mathrm{~h} \mathrm{10/30} \mathrm{gel-}$ filtration column (GE Healthcare) that had been preequilibrated in buffer containing $20 \mathrm{mM}$ Tris- $\mathrm{HCl} \mathrm{pH} 8.0$ and $150 \mathrm{mM} \mathrm{NaCl}$. The Acta chromatography system (GE Healthcare) was coupled to a MALS detector (mini-DAWN Treos) and a refractive index detector (Optilab DSP) (both from Wyatt Technology).

\section{Results}

\section{Crystal structure of caspase-8 tandem DEDs revealed domain-swapped dimerization}

In an effort to shed light on the mechanism of DISC assembly via oligomerization of caspase- 8 , FADD, death 
a $\begin{array}{lr}\text { CASPASE-8 } & 1 \\ \text { CASPASE-10 } & 1 \\ \text { CFLIP } & 1 \\ \text { CASPASE-8 } & 44 \\ \text { CASPASE-10 } \\ \text { CFLIP } \\ \\ \text { CASPASE-8 } \\ \text { CASPASE-10 } \\ \text { CFLIP } \\ \\ \text { CASPASE-8 } \\ \text { CASPASE-10 } \\ \text { CFLIP }\end{array}$

b

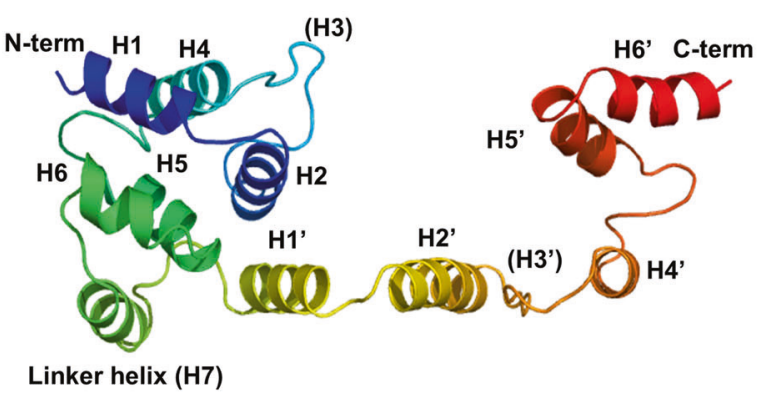

C

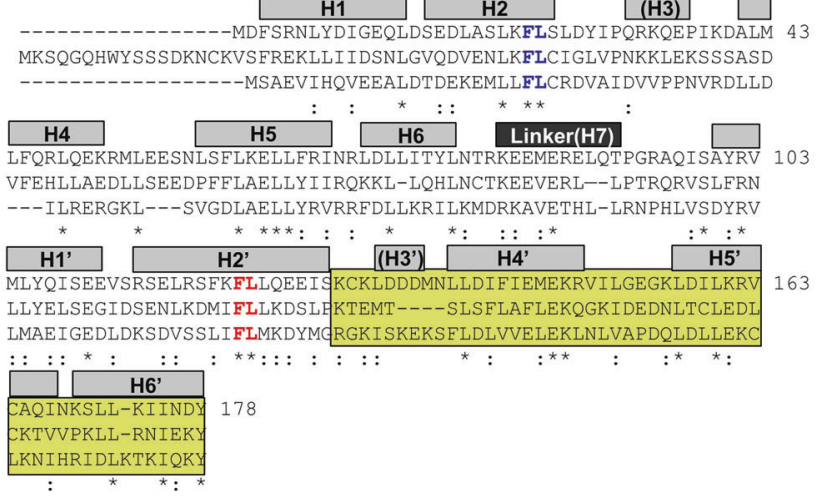

d

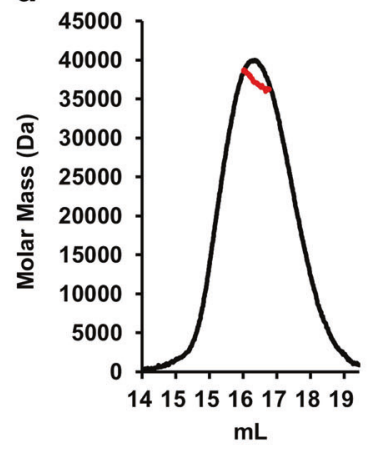

Fig. 1 The crystal structure of the tandem DEDs of wild-type caspase8. a Sequence alignment of tandem DEDs. Functionally important conserved FL motif on DED2 and another FL motif on DED1 are shown in red and blue color, respectively. Secondary structures that were observed in previous studies are indicated above the corresponding sequences (H1-H6 for DED1 and $\mathrm{H}^{\prime}-\mathrm{H}^{\prime}{ }^{\prime}$ for DED2). Partially unfolded region in DED2 due to the formation of a domainswapped dimer is highlighted in yellow color. $\mathbf{b}$ An illustration of the caspase- 8 tandem DED structure. The structure from the $\mathrm{N}$ terminus

receptor, and their specific interaction, we determined the crystal structure of the tandem DEDs (DED1-DED2) of caspase- 8 at $2.1 \AA$ resolution (Fig. $1 \mathrm{~b}$ and Table S1). The structure of the F122G/L123G double mutant form of caspase-8 (PDBid: 5JQE) [17], which has been designed for increased solubility, was used as a search model for MR. Our intact tandem DEDs of caspase- 8 were insoluble in solution as previously reported [17]. However, we successfully purified soluble caspase- 8 tandem DEDs by collecting tiny soluble fractions produced from $2 \mathrm{~L}$ bacterial cell cultures. We repeated small-scale $2 \mathrm{~L}$ purification steps 50 times to obtain $3 \mathrm{mg}$ of soluble caspase- 8 tandem DEDs (Fig. 1c). The structure was refined to an $R_{\text {work }}=22.0 \%$ and $R_{\text {free }}=26.9 \%$. The data collection and refinement statistics are summarized in Table S1. During the model-building step, we realized that one molecule was located in the asymmetric unit and the second DED part of the molecule was partially unfolded and extended to the opposite direction (Fig. 1b). The high-resolution structure of the intact $\begin{array}{lllll}670 & 158 & 44 & 17 & 1.35 \\ \mathbf{n D a}\end{array}$

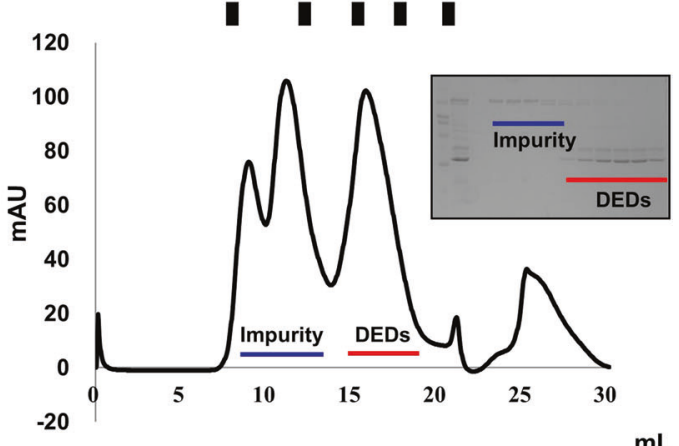

( $\mathrm{N}$-term) to the $\mathrm{C}$ terminus (C-term) is colored blue to red. Secondary structures of $\alpha$-helices are labeled in H1-H6 (DED1), H7 (linker), and H1'-H6' (DED2). c Tandem DED of caspase-8 was detected as a dimer in solution. The profile of size-exclusion chromatography. Among the three eluted peaks, the last peak eluted around $16-17 \mathrm{~mL}$ was a tandem DED based on SDS-PAGE (indicated as red bar). The other two peaks that eluted earlier were impurities (indicated as blue bar). d MALS profile. Red line indicates the experimentally calculated molecular weight

caspase- 8 tandem DEDs revealed that it was comprised of 13 helices, H1 to H6 for DED1, H7 for linker helix, and H1' to H6' for DED2 (Fig. 1b). A typical six-helical bundle fold of DED1 formed an eight helical bundle with linker helix $\mathrm{H} 7$ and $\mathrm{H}^{\prime}$ ' from DED2. H2'-H6' of DED2 were completely unfolded and extended to the opposite direction of DED1 (Fig. 1b). Previous biochemical and structural studies showed that tandem DEDs of caspase- 8 and its mutants could exist as monomers, dimers, or even higher oligomeric forms in solution $[17,25]$. To confirm the stoichiometry of caspase- 8 tandem DEDs in solution, we conducted sizeexclusion chromatography followed by MALS (SECMALS). Tandem DEDs of caspase- 8 were eluted at around $16 \mathrm{~mL}$, which corresponded to $\sim 40 \mathrm{kDa}$, indicating that it existed as a dimer in solution (Fig. 1c). This result was confirmed by MALS. The theoretically calculated molecular weight of caspase- 8 tandem DEDs with a C-terminal Histag was $18.41 \mathrm{kDa}$, and the experimental molecular weight from MALS was $38.19 \mathrm{kDa}$ (1.72\% fitting error), with a 
a

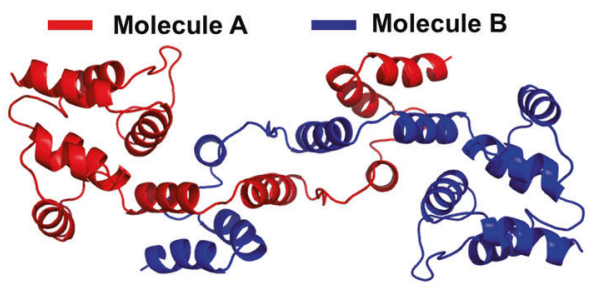

C

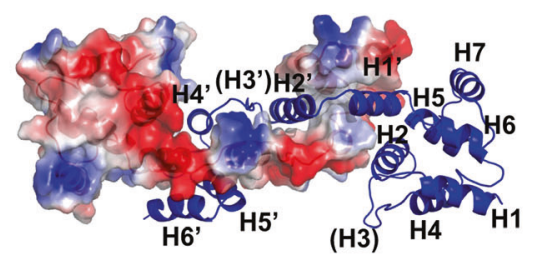

d

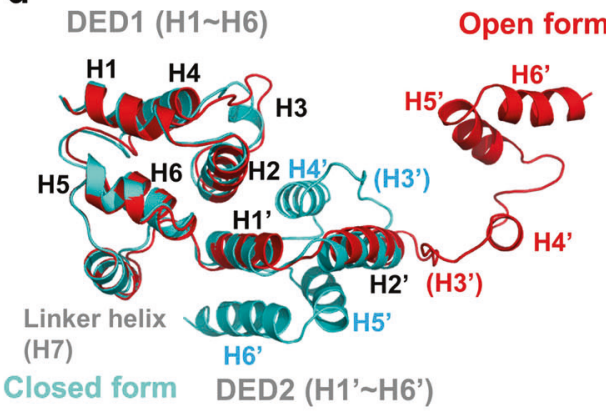

b
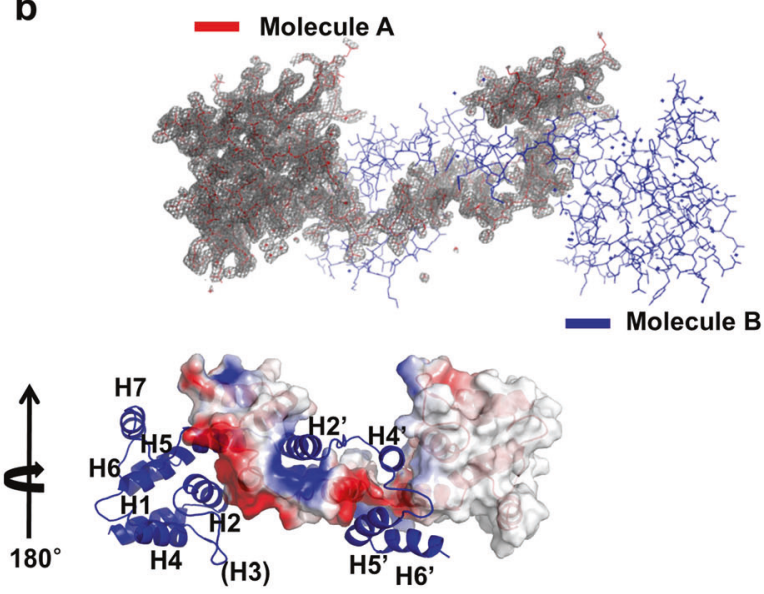

Molecule A Molecule B

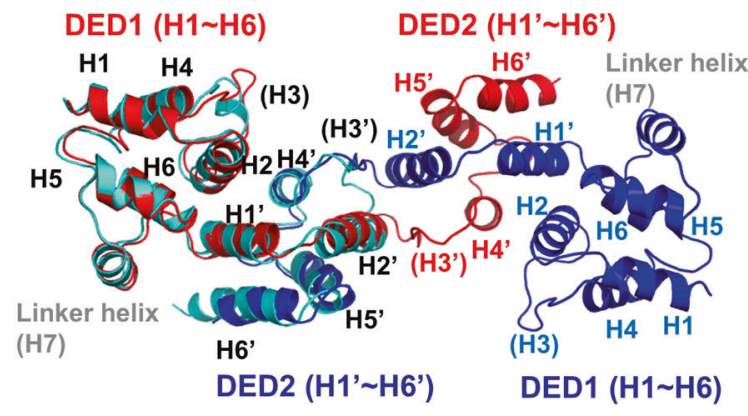

Fig. 2 Domain-swapped dimer of the caspase- 8 tandem DEDs. a Structure of the domain-swapped dimer. b $2 \mathrm{Fo}-\mathrm{Fc}$ electron density map contoured at $1.0 \sigma$ of molecule A. $\mathbf{c}$ Representation of electrostatic surface of caspase- 8 tandem DEDs showing the interface of domainswapped dimer. Secondary structures, from $\mathrm{H} 1$ to $\mathrm{H} 7$ (DED1) and H1' to $\mathrm{H}^{\prime}$ (DED2), are indicated on the corresponding helixes.

polydispersity of 1.0 (Fig. 1d). Based on our analysis using SEC-MALS, we concluded that tandem DEDs of caspase-8 existed as dimers in solution.

By searching for the symmetry molecule, second molecule, which interacts with the first molecule by domain swapping, was found (Fig. 2a). The electron density clearly showed that the helix bundle fold of the second DED (DED2) of caspase-8 was partially unfolded and used for domain swapping (Fig. 2b). Surface representation showed that the extended DED2 was well-fitted in the surface cleft formed by the extended DEDs from the opposite molecule (Fig. 2c). When compared to the monomeric structure of the caspase-8 FL motif mutant DED (F122G/L123G double mutant, PDBid: 5JQE), dislocation of the helix bundle fold started from H3' to H6' (Fig. 2d). For the domain swapping, displaced $\mathrm{H}^{\prime}, \mathrm{H}^{\prime}$, and $\mathrm{H}^{\prime}$ 'of molecule A were placed to another molecule (molecule B) to form a complete six helix bundle fold with $\mathrm{H}^{\prime}, \mathrm{H}^{\prime}$, and $\mathrm{H}^{\prime}$ of molecule $\mathrm{B}$, which is the typical structure of the death domain superfamily, including DED (Fig. 2d). d Structural comparison with monomeric, closed form of caspase-8 tandem DEDs. Current wild-type structure (red color) was superimposed with previously solved monomeric mutant structure (cyan color) (PDBid: 5JQE). e Structural comparison of dimeric caspase-8 tandem DED structure with monomeric, closed form of caspase-8 tandem DEDs

\section{The observed interface generated by domain swapping}

Procaspase-8 dimerized through domain swapping of caspase- 8 tandem DEDs (Fig. 3a-c). The interface was formed by massive $\mathrm{H}$-bonds and salt bridges (Fig. S1), which buried $\sim 3079 \AA^{2}$ among 13,017 $\AA^{2}$ total accessible surface area. PISA (Proteins, Interfaces, Structures and Assemblies) interface analyzing server scored 1.000 in the complex formation significance score, indicating that the domain-swapped dimer was a reasonable structure of the caspase-8 tandem DEDs. FL motif-containing helix at DED2 (H2'), in the middle of the domain-swapped dimer, mediated major interactions between the two molecules. Residue F122 on H2' formed a hydrophobic patch with F122 from the opposite molecule (Fig. 3b). Residue L133 on (H3 $\left.{ }^{\prime}\right)$ contributed to form this patch. Residue L133 on $\mathrm{H} 2^{\prime}$ formed another hydrophobic patch with residues V163 and $\mathrm{I} 167$ on H5' from the opposite molecule (Fig. 3b). The position of residues F122 and L123 were located in the 


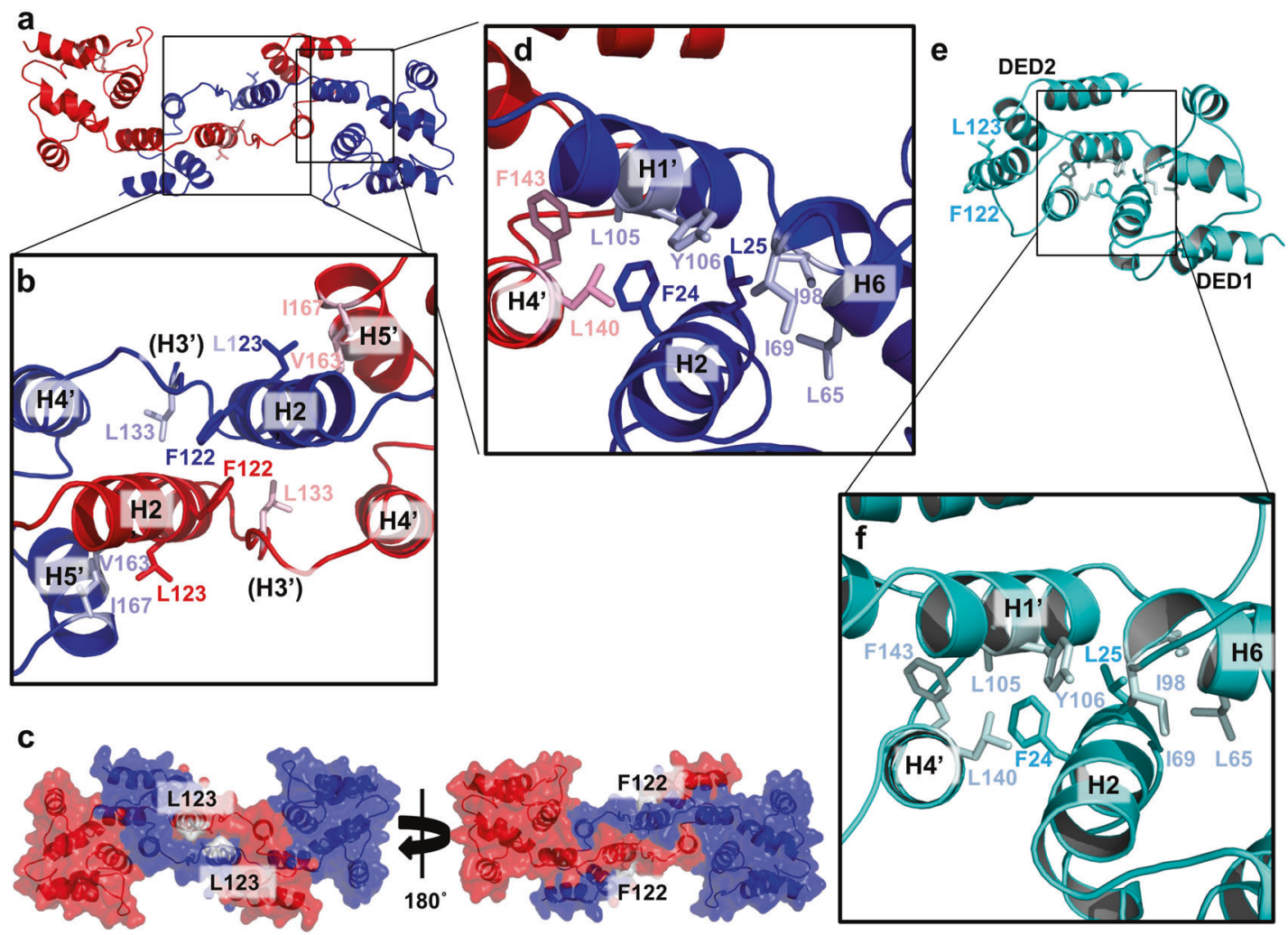

Fig. 3 The observed interface on the domain-swapped dimer of the caspase- 8 tandem DEDs. a The interface of the domain-swapped dimer. b, $\mathbf{c}$ Magnified view of the interface formed by the domainswapped dimer. Interaction interface formed in the middle (b) and side part (c) is magnified. d The monomeric structure of the FL motif mutant DED of caspase-8 (F122G/L123G double mutant, PDBid:
5JQE). The mutated FL motif is labeled. e Magnified view of the intramolecular interaction interface formed by two DEDs (DED1 and DED2) of caspase-8. f The location of the FL motif on DED2, which is critical for the dimerization-mediated activation of caspase-8, on the surface of the domain-swapped dimer of caspase- 8 tandem DEDs middle of the interface surface formed by the domainswapped dimer (Fig. 3c). These residues, F122 and L123 on DED2, were largely conserved in other tandem DEDcontaining proteins (Fig. 1a). FL motif on DED1 was involved in the formation of intermolecular hydrophobic patches with neighboring hydrophobic residues, including L105 and Y106 on H1', and L65 and I69 on H6. Dislocated H4' by domain swapping was re-located in between H1' and $\mathrm{H} 2$ of the opposite molecule and was involved in the hydrophobic interaction (Fig. 3d). According to the monomeric mutant tandem DEDs of caspase-8, FL motif on DED1 was located inside of the tandem DEDs and mediated formation of the hydrophobic core formed in between DED1 and DED2. In contrast, FL motif on DED2 was located at the end of one side portion on the surface (Fig. 3e, f). The hydrophobic core, which was formed by the FL motif on $\mathrm{H} 2$ and other hydrophobic resides on H6, $\mathrm{H}^{\prime}$, and $\mathrm{H}^{\prime}{ }^{\prime}$ and is critical for the correct folding of the caspase- 8 tandem DED structure by stabilized DED1 and DED2, was the same as that detected at the domainswapped dimer. $\mathrm{H}^{\prime}$ from the same molecule was replaced by $\mathrm{H}^{\prime}$ ' from the opposite molecule by domain swapping (Fig. 3d-f).

Beside the hydrophobic cores formed by FL motifs on DED1 and DED2, massive salt bridges and H-bonds were newly formed through domain swapping. R102, R118, E127, D135, E147, and R162 from molecule A formed salt bridges with the same residues as the oppositely located molecule B (Fig. S1). This combination of massive interactions made the dimeric complex stable and lowered Bfactors (Fig. S2).

\section{FL motif on DED2 is critical for caspase-8 dimerization via domain swapping}

The importance of the FL motif on DED2 for dimerization, oligomerization, and further activation of caspase- 8 has been reported by various groups $[16,18,26]$. Additionally, FL motif mutation to glycine or alanine increased solubility of caspase- 8 tandem DEDs and improved possibility for structural studies $[17,25]$. To determine why the FL motif on DED2 is critical for caspase-8 function, we investigated our 

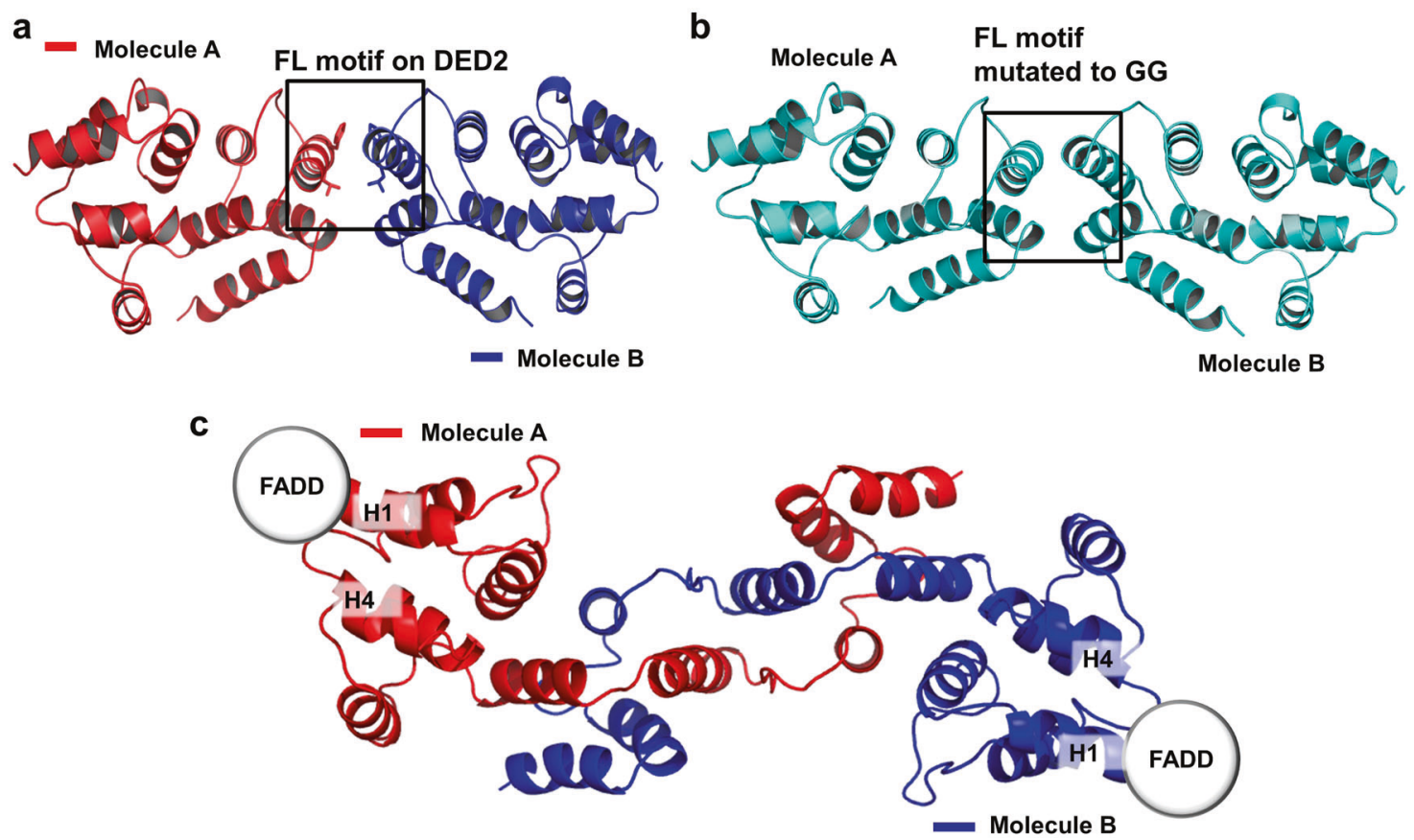

Fig. 4 FL motif on DED2 is critical for caspase-8 dimerization via domain swapping. a The location of the FL motif on DED2. Closely located FL motif on two different tandem DEDs from caspase- 8 are boxed. b The structure of FL motif mutants of caspase- 8 tandem

structure. Since the FL motif on DED2 mediated domain swapping, it might be feasible that two closely located FL motifs on DED2 may trigger domain swapping with two different caspase-8 via strong hydrophobic interactions (Fig. 4a). However, mutation of the FL motif on DED2 to either alanine or glycine lost its capability to interact with each other to trigger domain swapping (Fig. 4b).

Although DED2 of caspase-8 was suggested to bind with the FADD adaptor for DISC assembly [7, 27], recent biochemical and computational studies indicated that FADD binds to the $\mathrm{H} 1$ and $\mathrm{H} 4$ regions on DED1 of caspase- 8 $[15,16,18,26]$, which is supported by our structure. Because FL motif and most on the helices on DED2 are involved in the domain swapping-mediated homodimerization, FADD should bind to available $\mathrm{H} 1$ and $\mathrm{H} 4$ regions on DED1 of caspase-8 (Fig. 4c and Fig. S3).

\section{A model of DISC assembly followed by caspase-8 activation via dimerization}

Based on our novel dimer structure of caspase- 8 , a model of DISC assembly followed by caspase- 8 activation was proposed. Caspase activation may proceed in several steps (Fig. 5a). At a basal state, without recruiting FADD to the death receptor, monomeric caspase- 8 is freely available in the cell. Upon apoptosis induction triggered by interaction of the death ligand to the trimeric death receptor (steps 1 and 2), adaptor FADD is recruited to the death receptor via a DD-DD
DEDs. Closely located mutant FL motif on two different tandem DEDs from caspase- 8 are boxed. $\mathbf{c}$ Tentative interaction mode between caspase- 8 tandem DEDs and FADD DED. The position of helix1 and helix 4 on caspase-8 DED1 is labeled as $\mathrm{H} 1$ and $\mathrm{H} 4$

interaction (step 3). Subsequently, FADD recruited at cell surface can recruit caspase-8 via a DED-DED interaction (step 4), leading to procaspase-8 proximity to meet another procaspase- 8 . This DISC assembly mediates accessibility of FL motif on procaspase-8 DED2 (step 5). Closely located FL motif on DED2 causes domain swapping and dimerization of procaspase-8 (step 6). The dimerization via tandem DED domain swapping causes the proximity-mediated self-activation of caspase-8. Because trimeric death receptor association is known to be critical for DISC assembly, the complete massive DISC complex is expected to form a cluster in lipid rafts (Fig. 5b, c).

This new model derived from our novel structure can explain why FL motif on DED2 is critical for the dimerization, oligomerization, and activation of caspase-8. In addition, this model can also explain the increased local concentration of initiator caspase by DISC formation, which is critical for the activation of initiator caspase.

\section{Discussion}

Caspases are a family of cysteine proteases that work in the apoptosis and inflammation signaling pathways. Caspases are divided into two classes, initiator and effector, and caspase- 8 is a representative initiator caspase. Initiator caspases are auto-activated when they obtain proximity through recruitment to oligomeric signaling complexes. For 
a

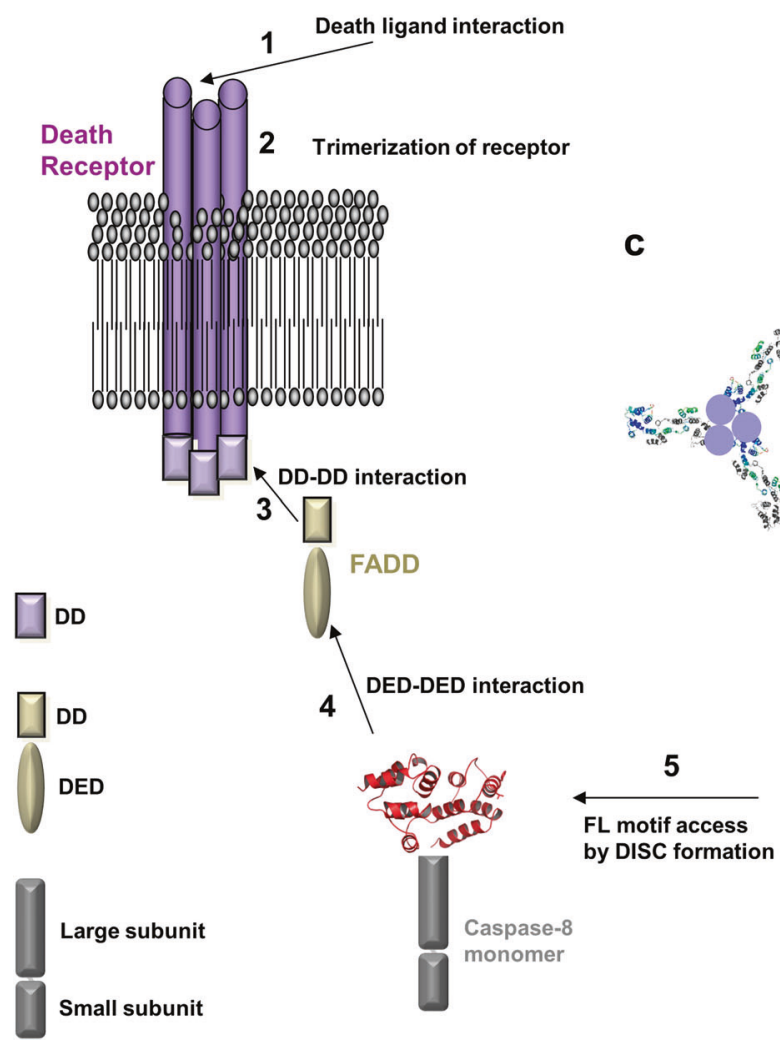

Fig. 5 A model of DISC assembly followed by dimerization-mediated caspase- 8 activation. a Proposed procedure of caspase- 8 activation via domain-swapped dimerization. b, $\mathbf{c}$ Massive DISC chain assembly via

the activation of caspase-8, DISC, which is composed of Fas, FADD, and caspase- 8 , is formed to recruit caspase-8. Caspase- 8 contains pro-domains at the $\mathrm{N}$ terminal, also called tandem DEDs, which are critical for DISC formation and the protein interaction.

Although intensive studies have been performed during several decades, the recruitment process of initiator caspase in DISC and further activation is still controversial. To unveil how caspase- 8 is adapted at the trimeric death receptor, oligomerized, and further activated by gaining proximity, we determined the structure of intact tandem DEDs of caspase-8. Surprisingly, our structure revealed novel domain-swapped dimerization that has not been reported previously for any death domain superfamily, including other DEDs despite extensive structural pursuit. Caspase- 8 dimerization is known to be critical for activation. Domain-swapped dimerization strategy detected in current study can explain why the FL motif on DED2 is critical for dimerization, further oligomerization, and activation of caspase- 8 . Our structure can also explain the increased local concentration-dependent activation of initiator caspase. For the domain swapping of tandem DEDs and further activation, two procaspase- 8 have to be closely
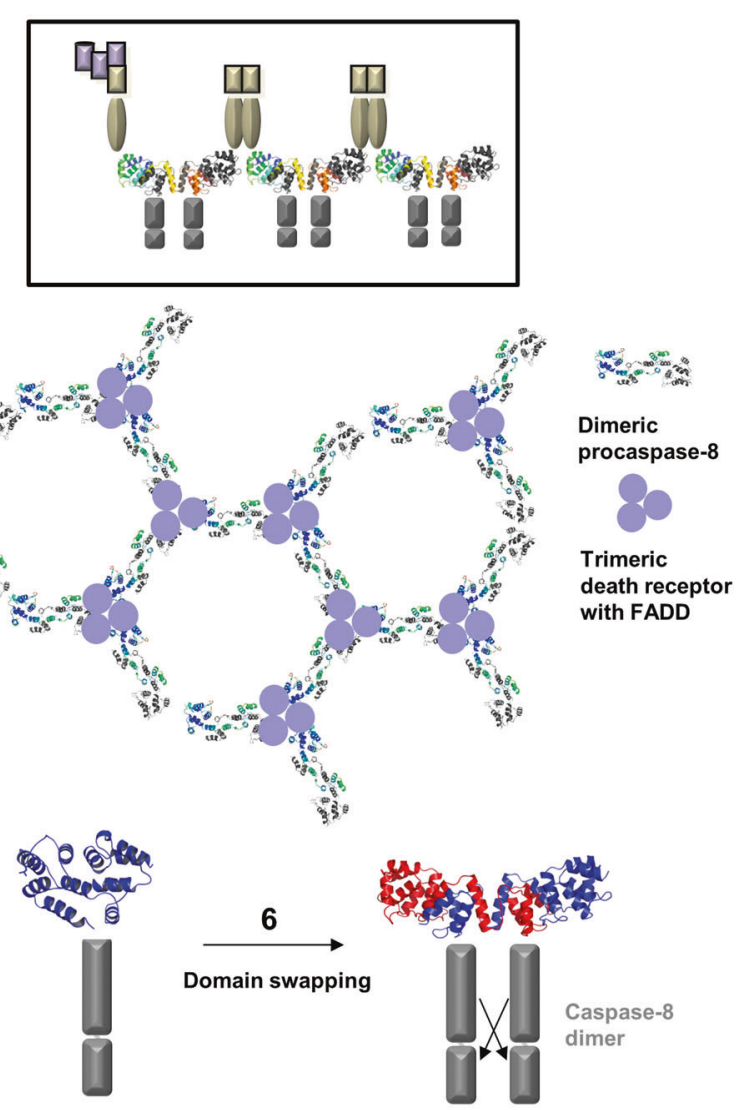

interaction of the trimeric death receptor, adaptor FADD, and domainswapped caspase-8. Side view (b) and top view (c) are proposed

related. FADD-binding site on the caspase- 8 tandem DEDs, which is known to be the $\mathrm{H} 1$ and $\mathrm{H} 4$ regions of DED1, is also explained with our structure. Therefore, our studies uncovered a novel tandem DED-mediated dimerization mechanism, which is critical for DISC assembly followed by initiator caspase activation.

Taken together, our domain-swapped dimer structure of caspase- 8 tandem DEDs is the first structure of unmodified wild-type caspase-8 DEDs, which can explain dimerization and further cluster formation using the FL motif on DED2 as critical for oligomerization. Because caspase- 8 can form many different oligomeric complexes with various signaling molecules during apoptosis, necroptosis, and other immune signaling pathways by acting as an enzyme and sometimes scaffolding adaptor $[12,28]$, it is not surprising that several different forms of procaspase-8, including current domainswapped dimer form and previously reported filament form [17], can be found in the cell. A similar case was also detected in the study of Fas DD and FADD DD complexes, whose structures were reported as two different forms, helical oligomeric complex [29] and structurally altered Fas DD-mediated complex [30]. Structural alteration of DD superfamily, including $\mathrm{DD}$ and $\mathrm{DED}$, might regulate 
assembly of homo- or hetero-oligomeric complexes in cellular signaling. The controlling points and effectors that can determine the different forms of DD superfamily complexes, especially caspase- 8 tandem DEDs during various signaling events, must be studied further in the future.

\section{Accession number}

Atomic coordinates and structure factors have been deposited in the Protein Data Bank with PDB ID: 6AGW.

Acknowledgements I thank the staff at BL-5C of the Pohang Accelerator Laboratory (Pohang, Korea) for their kind help with data collection. This study was supported by the National Research Foundation of Korea (NRF) grant funded by the Korea government (MSIT) (NRF-2017M3A9D8062960, NRF-2018R1A2B2003635, and NRF-2018R1A4A1023822) and a grant from the Korea Healthcare Technology R\&D Project, Ministry of Health \& Welfare, Republic of Korea (HI17C0155).

Author contributions HHP designed the project, performed experiments, and wrote the manuscript.

\section{Compliance with ethical standards}

Conflict of interest The author declares that he has no conflict of interest.

\section{References}

1. Denault JB, Salvesen GS. Caspases: keys in the ignition of cell death. Chem Rev. 2002;102:4489-500.

2. Hardy JA, Lam J, Nguyen JT, O'Brien T, Wells JA. Discovery of an allosteric site in the caspases. Proc Natl Acad Sci USA. 2004;101:12461-6.

3. Salvesen GS, Dixit VM. Caspases: intracellular signaling by proteolysis. Cell. 1997;91:443-6.

4. Salvesen GS, Dixit VM. Caspase activation: the inducedproximity model. Proc Natl Acad Sci USA. 1999;96:10964-7.

5. Kischkel FC, Hellbardt S, Behrmann I, Germer M, Pawlita M, Krammer PH, et al. Cytotoxicity-dependent APO-1 (Fas/CD95)associated proteins form a death-inducing signaling complex (DISC) with the receptor. EMBO J. 1995;14:5579-88.

6. Park HH, Lo YC, Lin SC, Wang L, Yang JK, Wu H. The death domain superfamily in intracellular signaling of apoptosis and inflammation. Annu Rev Immunol. 2007;25:561-86.

7. Carrington PE, Sandu C, Wei Y, Hill JM, Morisawa G, Huang T, et al. The structure of FADD and its mode of interaction with procaspase-8. Mol Cell. 2006;22:599-610.

8. Boatright KM, Renatus M, Scott FL, Sperandio S, Shin H, Pedersen IM, et al. A unified model for apical caspase activation. Mol Cell. 2003;11:529-41.

9. Christofferson DE, Yuan J. Necroptosis as an alternative form of programmed cell death. Curr Opin Cell Biol. 2010;22:263-8.

10. Gunther C, Martini E, Wittkopf N, Amann K, Weigmann B, Neumann H, et al. Caspase-8 regulates TNF-alpha-induced epithelial necroptosis and terminal ileitis. Nature. 2011;477:335-9.

11. Su H, Bidere N, Zheng L, Cubre A, Sakai K, Dale J, et al. Requirement for caspase-8 in NF-kappaB activation by antigen receptor. Science. 2005;307:1465-8.
12. Chun HJ, Zheng L, Ahmad M, Wang J, Speirs CK, Siegel RM, et al. Pleiotropic defects in lymphocyte activation caused by caspase- 8 mutations lead to human immunodeficiency. Nature. 2002;419:395-9.

13. Donepudi M, Mac Sweeney A, Briand C, Grutter MG. Insights into the regulatory mechanism for caspase- 8 activation. Mol Cell. 2003;11:543-9.

14. Muzio M, Stockwell BR, Stennicke HR, Salvesen GS, Dixit VM An induced proximity model for caspase- 8 activation. J Biol Chem. 1998;273:2926-30.

15. Riley JS, Malik A, Holohan C, Longley DB. DED or alive: assembly and regulation of the death effector domain complexes. Cell Death Dis. 2015;6:e1866.

16. Dickens LS, Boyd RS, Jukes-Jones R, Hughes MA, Robinson GL, Fairall L, et al. A death effector domain chain DISC model reveals a crucial role for caspase- 8 chain assembly in mediating apoptotic cell death. Mol Cell. 2012;47:291-305.

17. Fu TM, Li Y, Lu A, Li Z, Vajjhala PR, Cruz AC, et al. Cryo-EM structure of caspase- 8 tandem DED filament reveals assembly and regulation mechanisms of the death-inducing signaling complex. Mol Cell. 2016;64:236-50.

18. Majkut J, Sgobba M, Holohan C, Crawford N, Logan AE, Kerr E, et al. Differential affinity of FLIP and procaspase 8 for FADD's DED binding surfaces regulates DISC assembly. Nat Commun. 2014;5:3350.

19. Schleich K, Warnken U, Fricker N, Ozturk S, Richter P, Kammerer K, et al. Stoichiometry of the CD95 death-inducing signaling complex: experimental and modeling evidence for a death effector domain chain model. Mol Cell. 2012;47:306-19.

20. Otwinowski Z, Minor W. Processing of X-ray diffraction data collected in oscillation mode. Methods Enzymol. 1997;276:307-26.

21. McCoy AJ. Solving structures of protein complexes by molecular replacement with Phaser. Acta Crystallogr D Biol Crystallogr. 2007;63(Pt 1):32-41.

22. Emsley P, Cowtan K. Coot: model-building tools for molecular graphics. Acta Crystallogr D Biol Crystallogr. 2004;60(Pt 12 Pt 1):2126-32.

23. Adams PD, Afonine PV, Bunkoczi G, Chen VB, Davis IW, Echols N, et al. PHENIX: a comprehensive Python-based system for macromolecular structure solution. Acta Crystallogr D Biol Crystallogr. 2010;66(Pt 2):213-21.

24. Laskowski RA, MacArthur MW, Moss DS, Thornton JM. PROCHECK: a program to check the stereochemical quality of protein structures. J Appl Cryst. 1993;26:283-91.

25. Shen C, Yue H, Pei J, Guo X, Wang T, Quan JM. Crystal structure of the death effector domains of caspase-8. Biochem Biophys Res Commun. 2015;463:297-302.

26. Singh N, Hassan A, Bose K. Molecular basis of death effector domain chain assembly and its role in caspase- 8 activation. FASEB J. 2016;30:186-200.

27. Kersse K, Verspurten J, Vanden Berghe T, Vandenabeele P. The death-fold superfamily of homotypic interaction motifs. Trends Biochem Sci. 2011;36:541-52.

28. Wang L, Du F, Wang X. TNF-alpha induces two distinct caspase8 activation pathways. Cell. 2008;133:693-703.

29. Wang L, Yang JK, Kabaleeswaran V, Rice AJ, Cruz AC, Park AY, et al. The Fas-FADD death domain complex structure reveals the basis of DISC assembly and disease mutations. Nat Struct Mol Biol. 2010;17:1324-9.

30. Scott FL, Stec B, Pop C, Dobaczewska MK, Lee JJ, Monosov E, et al. The Fas-FADD death domain complex structure unravels signalling by receptor clustering. Nature. 2009;457: 1019-22. 